\title{
Terahertz Emission Driven by Two-color Laser Pulses at Various Frequency Ratios
}

\author{
W.-M. Wang, ${ }^{1,2}$ Z.-M. Sheng, ${ }^{3,4,5}$ Y.-T. Li, ${ }^{1,5,6}$ Y. Zhang, ${ }^{2}$ and J. Zhang ${ }^{3,5}$ \\ ${ }^{1}$ Beijing National Laboratory for Condensed Matter Physics, \\ Institute of Physics, CAS, Beijing 100190, China \\ ${ }^{2}$ Beijing Advanced Innovation Center for Imaging Technology, \\ Department of Physics, Capital Normal University, Beijing 100048, China \\ ${ }^{3}$ Key Laboratory for Laser Plasmas (MoE) and Department of Physics and Astronomy, \\ Shanghai Jiao Tong University, Shanghai 200240, China \\ ${ }^{4}$ SUPA, Department of Physics, University of Strathclyde, \\ Glasgow G4 ONG, United Kingdom \\ ${ }^{5}$ IFSA Collaborative Innovation Center, \\ Shanghai Jiao Tong University, Shanghai 200240, China \\ ${ }^{6}$ School of Physical Sciences, University of Chinese \\ Academy of Sciences, Beijing 100049, China
}

(Dated: August 4, 2017)

\begin{abstract}
We present a simulation study of terahertz radiation from a gas driven by two-color laser pulses in a broad range of frequency ratios $\omega_{1} / \omega_{0}$. Our particle-in-cell simulation results show that there are three series with $\omega_{1} / \omega_{0}=2 n, n+1 / 2, n \pm 1 / 3$ ( $n$ is a positive integer) for high-efficiency and stable radiation generation. The radiation strength basically decreases with the increasing $\omega_{1}$ and scales linearly with the laser wavelength. These rules are broken when $\omega_{1} / \omega_{0}<1$ and much stronger radiation may be generated at any $\omega_{1} / \omega_{0}$. These results can be explained with a model based on gas ionization by two linear-superposition laser fields, rather than a multiwave mixing model.
\end{abstract}

PACS numbers: 42.65.Re, 32.80.Fb, 52.38.-r, 52.65.Rr 


\section{INTRODUCTION}

Strong terahertz $(\mathrm{THz})$ waves have broad applications in physics [1-4], biology [5] and medicine [6]. A large number of studies have shown that interactions of intense fs laser pulses with plasmas can provide powerful table-top $\mathrm{THz}$ sources with tunable parameters. Near mJ level of $\mathrm{THz}$ radiation with large divergence and a band from $0.1 \mathrm{THz}$ to $100 \mathrm{THz}$ [7-12] has been observed from solid targets interacted by relativistic intense laser pulses. Nearly linearly-polarized, single-cycle, $\mathrm{MV} / \mathrm{cm}$-scale $\mathrm{THz}$ radiation with good directionality [13-27] has been generated from a gas irradiated by weakly relativistic intense laser pulses, via the well-known two-color laser scheme in which a fundamental laser pulse is mixed with a second pulse at the second harmonic [13]. With circularly-polarized or ellipticallypolarized driving laser pulses [28-30], the radiation can become elliptically-polarized. When an external magnetic field is imposed in the two-color scheme with linearly-polarized laser pulses, the radiation can be narrow-band, circularly-polarized [31].

To further achieve more powerful $\mathrm{THz}$ radiation, a few schemes were proposed by increasing the laser wavelength. In 2011, a mid-infrared laser scheme was proposed to significantly enhance the radiation strengths [32], because the ionization symmetry in a laser cycle can be broken more strongly with a longer wavelength laser pulse. The subsequent experiments $[33,34]$ showed that mid-infrared laser pulses are more favorable for $\mathrm{THz}$ generation than conventional $0.8 \mu \mathrm{m}$ wavelength pulses. In particular, a strong dependence of $\mathrm{THz}$ generation on the laser wavelength in the two-color laser scheme was found $[34,35]$ and $4.4 \mathrm{MV} / \mathrm{cm}$ $\mathrm{THz}$ radiation was generated in experiments with a $1.8 \mu \mathrm{m}$ wavelength pulse and its second harmonic pulse [34].

A few of recent works showed that the two-color laser scheme can be extended. In 2013, a model based on linear superposition of two laser fields predicted [36] that as long as the frequency ratio $\omega_{1} / \omega_{0}$ of the two laser pulses is $2 n$ ( $n$ is a positive integer), THz radiation can be generated, which was verified by particle-in-cell (PIC) simulations. In 2016, Kostin et al. [37] performed detailed calculations with $\omega_{1} / \omega_{0}$ from 0.4 to 3 and found THz radiation can be generated when $\omega_{1} / \omega_{0}$ are $2 / 3,3 / 2$, etc. They proposed a multiwave mixing theory to well explain their calculation results. In addition, Gonzalez de Alaiza Martinez et al. [38] proposed that if the two-frequency pulses are extended to multiple-frequency pulses with fixed phases and strengths for each pulses (it is a sawtooth wave in the limit case), the $\mathrm{THz}$ 
strength can be significantly increased.

In this paper, we further study the two-color laser scheme with broader frequency ratios to generate $\mathrm{THz}$ radiation. When the frequency ratio $\omega_{1} / \omega_{0}$ is limited within 0.4 and 3 , our PIC simulation results agree with the calculation results in Ref. [37], which can be described by the multiwave mixing theory. However, when the region of $\omega_{1} / \omega_{0}$ is extended to be below 0.4 , the predictions by the multiwave mixing theory contradict with our PIC results. Contrary to the predictions [37], the $\mathrm{THz}$ radiation could be generated at any $\omega_{1} / \omega_{0}=a: b$ ( $a$ and $b$ are natural), even if $a+b$ is even; the upper-limit of $a+b$ for the $\mathrm{THz}$ generation does not depend on the laser intensity; and the $\mathrm{THz}$ generation is not necessarily weakened with the growing $a+b$, e.g., the radiation with $\omega_{1} / \omega_{0}=3 / 10$ can be stronger than the one with $\omega_{1} / \omega_{0}=3 / 2$. These results can be explained by gas ionization by two linear-superposition laser fields, suggesting that the multiwave mixing theory might not reflect the physical insights.

We find that when $\omega_{1} / \omega_{0}$ is $2 n, n+1 / 2, n \pm 1 / 3$ ( $n$ is a positive integer), the $\mathrm{THz}$ generation is highly efficient and stable against the changing laser duration and intensity, where the $2 n$ series has the best performance. The THz strength basically decreases with the increasing $n$ and scales linearly with the laser wavelength in the tunneling ionization regime. With this linear wavelength scaling, one can link the $\mathrm{THz}$ generation in the two cases between $\omega_{1} / \omega_{0}=2 / 3$ and $3 / 2$, or between $\omega_{1} / \omega_{0}=1 / 2$ and 2 , or between $\omega_{1} / \omega_{0}=1 / 4$ and 4 , etc. These simulation results are explained by a model based on gas ionization by two linear-superposition laser fields.

\section{THZ GENERATION WITH VARIOUS FREQUENCY RATIOS}

\section{A. Setup of PIC simulation}

We first present results of PIC simulations with the two-dimensional (2D) KLAPS code [39] and then explain the PIC results by a theoretic model. In the PIC code [39], the field ionization of gases is included and the full Maxwell equations are be used to calculate electromagnetic field generation and propagation. Therefore, it can self-consistently calculate the plasma production and net current formation via ionization by the incident laser pulses, dynamics of the net current in the plasma, and $\mathrm{THz}$ radiation generation. Note that the dis- 
persion and absorption of electromagnetic waves in formed plasma is taken into account, but the dispersion and absorption in a gas is not included, which could be ignored in a near-field case or a short distance of propagation. In the current study, the laser wavelength/cycle needs to be resolved since the $\mathrm{THz}$ generation depends on relative phase between the twocolor pulses. Thus, it is difficult to compute a long distance of propagation (e.g., millimeter and centimeter scales) of laser and $\mathrm{THz}$ waves by PIC simulation. $\mathrm{A}(3+1)$-dimensional simulation approach proposed by Babushkin et al. [40,41] can effectively calculate a long distance of propagation, based on a unidirectional pulse propagation equation, which can obtain far-field THz emission. On the other hand, the PIC approach is suitable to calculate the near-field $\mathrm{THz}$ radiation generation, which determines the main properties (frequency, waveform, strength, polarization, etc.) of far-field $\mathrm{THz}$ radiation observed in experiments, since the final far-field radiation is composed of many near-field sources [27, 41]. Hence, the PIC simulations can self-consistently estimate $\mathrm{THz}$ generation by two-color laser pulses at various frequency ratios. Note that in Ref. [37] the gas ionization is calculated, but the $\mathrm{THz}$ generation is not included.

In our PIC simulations, the frequency $\omega_{0}$ of the first laser pulse is fixed with the wavelength $\lambda_{0}=0.8 \mu \mathrm{m}$ (or the period $\tau_{0}=2.667 \mathrm{fs}$ ) and the second laser frequency $\omega_{1}$ is changed in the range from $0.1 \omega_{0}$ to $20 \omega_{0}$. The two pulses propagate along the $+x$ direction with linear polarization along the $y$ direction. They have the same spot radius $r_{0}=100 \mu \mathrm{m}$, duration $50 \mathrm{fs}$ in full width at half maximum (FWHM), and peak intensity $I_{0}=I_{1}=10^{14} \mathrm{~W} / \mathrm{cm}^{2}$ (or peak strengths $274 \mathrm{MV} / \mathrm{cm}$ ), as used in Ref. [37]. The total laser energy is $1.86 \mathrm{~mJ}$. The laser electric fields are taken as $\mathbf{E}=\vec{e}_{y}\left[a_{0} \sin \left(\omega_{0} \psi\right)+a_{1} \sin \left(\omega_{1} \psi+\theta_{1}\right)\right] \exp \left(-y^{2} / r_{0}^{2}\right) f(\psi)$, where $\psi=t-x / c, f(\psi)$ is the Gaussian envelope profile of the laser pulses, and the relative phase $\theta_{1}$ of the two pulses is zero (with $\theta_{1}=0$ the strongest radiation can be generated with $\omega_{1} / \omega_{0}=2 n, n+1 / 2, n \pm 1 / 3$ according to our simulation results). A hydrogen gas slab is taken with a length $300 \mu \mathrm{m}$ and a uniform density $4.88 \times 10^{16} \mathrm{~cm}^{-3}$ (the corresponding plasma frequency $\omega_{p} / 2 \pi=2 \mathrm{THz}$ after the complete ionization [36]). The resolutions along the $\mathrm{x}$ and $\mathrm{y}$ directions are $0.01 \lambda_{0}$ and $0.1 \lambda_{0}$, respectively. In the gas region, four simulation particles per cell are adopted to denote gas atoms. In all the simulations, the gas parameters and the laser relative phase are kept unchanged. In Fig. 4 and Table I we take the standard parameters mentioned above. Besides $\omega_{1}$, only one laser parameter is changed as addressed in figure legends: we used different laser durations in Fig. 1, different laser intensities in 
Figs. 2 and 3, and different $\omega_{0}$ in Fig. 5.

Note that we do not observe THz generation above $5 \mathrm{kV} / \mathrm{cm}$ when $\omega_{1}$ is taken as $12-20 \omega_{0}$. One can expect no THz generation with a higher value of $\omega_{1}$. For the case with $\omega_{1}<0.1 \omega_{0}$, the pulse of $50 \mathrm{fs}$ has the effective cycle number below 2. Therefore, the frequency ratio of 0.1-20 taken in our simulations should cover the whole range possibly adopted in laboratories currently.

\section{B. PIC simulation results}

Figure 1(a) displays absolute values of the $\mathrm{THz}$ radiation strengths observed in the left vacuum $5 \lambda_{0}$ away from the vacuum-gas boundary. The PIC simulation results in the range of $\omega_{1} / \omega_{0}$ from 0.4 to 3 are in agreement with the results by calculating the net current generation in Ref. [37], in which the investigation is limited in this frequency range (note that the THz strength is proportional to the net current strength $[19,42])$. Peaks of the THz strengths appear at $\omega_{1} / \omega_{0}=1 / 2,2 / 3,4 / 3,3 / 2,2,5 / 2$ (strength signs can be observed in Fig. 2 below), as given in Ref. [37]. As $\omega_{1} / \omega_{0}$ is reduced from 0.4 to 0.1, some new peaks arise with higher strengths than the peaks at $\omega_{1} / \omega_{0}>0.4$, as shown more clearly in Fig. 1(b). In particular, the strengths at $\omega_{1} / \omega_{0}=1 / 4,1 / 8,1 / 9,1 / 10$ exceed the strength $0.17 \mathrm{MV} / \mathrm{cm}$ at $\omega_{1} / \omega_{0}=2$ (the original two-color scheme [13]). This result contradicts with the multiwave mixing theory proposed in Ref. [37], which drew the conclusions as: to generate THz radiations, $a+b$ should be an odd integer $\left(\omega_{1} / \omega_{0}=a: b, a: b\right.$ is irreducible, and $a$ and $b$ are natural); and the radiation is weakened with the increasing $a+b$. These conclusions can summarize the results with $\omega_{1} / \omega_{0}$ between 0.4 and 3 . However, they are broken when $\omega_{1} / \omega_{0}$ is lower than 0.4 . Strong THz radiations can be generated even if $a+b$ is even, e.g., $\omega_{1} / \omega_{0}=1 / 9$. The strength with larger $a+b$ is not necessarily lower, e.g., comparing the result at $\omega_{1} / \omega_{0}=2 / 3$ with the ones at $\omega_{1} / \omega_{0}=1 / 9$ or $1 / 6$, etc.

One of the most important conclusions given by the multiwave mixing theory [37] is that $a+b$ should less than $m_{0}$ for the $\mathrm{THz}$ generation and $m_{0}$ is determined by the laser intensity, e.g., $m_{0}=11$ for $I_{0}=10^{14} \mathrm{~W} / \mathrm{cm}^{2}$ [37]. This does not agree with the results shown in Fig. 1(b) that the THz radiations can be generated at $\omega_{1} / \omega_{0}=1 / 10,3 / 10$ with $a+b \geq 11$. Furthermore, if the laser intensity is enhanced, $m_{0}$ should be greater than 11 according to the multiwave mixing theory. However, when we increase the laser intensity 
from $I_{0}=10^{14} \mathrm{~W} / \mathrm{cm}^{2}$ to $4 \times 10^{14} \mathrm{~W} / \mathrm{cm}^{2}$ (also $2 \times 10^{14} \mathrm{~W} / \mathrm{cm}^{2}$ ), we do not observe $\mathrm{THz}$ generation at any new point of $\omega_{1} / \omega_{0}$ with $a+b \geq 11$, as shown in Fig. 2. In this figure we only illustrate the results with $\omega_{1} / \omega_{0}$ at $2 n, n+\frac{1}{2}, n \pm \frac{1}{3}$ ( $n$ is a positive integer), at which the $\mathrm{THz}$ generation has high efficiency and good stability as will be addressed below. All in all, our simulations show that there is not a cutoff of $a+b$ dependent of laser intensity, required for the $\mathrm{THz}$ generation.

In addition, one can see in Fig. 1 that the THz generation is significantly affected by laser duration. In this figure, we slightly change the laser duration and observe sharp varieties in the $\mathrm{THz}$ strengths when $\omega_{1} / \omega_{0}<1 / 3$. Even the signs of the THz strengths is reversed at some $\omega_{1} / \omega_{0}$ values, when the laser duration is changed from 50 fs to 47 fs. When $\omega_{1} / \omega_{0}>1 / 3$, the THz generation slightly changes with the laser duration. In our additional simulations, we take the laser duration to $25 \mathrm{fs}$, change the duration around this value, and find the THz strength significantly changes when $\omega_{1} / \omega_{0}<0.6$. Once more, this cannot be explained by the multiwave mixing theory [37].

\section{EXPLANATIONS BY GAS IONIZATION OF TWO LINEAR-SUPERPOSITION LASER FIELDS}

From the simulation results above, one can see that although the multiwave mixing theory [37] can summarize most results of $\mathrm{THz}$ generation with $\omega_{1} / \omega_{0}$ within 0.4 and 3 , it is not consistent with the results with lower $\omega_{1} / \omega_{0}$. In the following, we will explain all the results through gas ionization by two linear-superposition laser fields.

We first explain why stronger $\mathrm{THz}$ radiation can be generated with the lower $\omega_{1} / \omega_{0}$, in particular $\omega_{1} / \omega_{0}=1 / 9,1 / 7,1 / 5$ with which the radiation should have not be generated. In Figs. 3(a)-3(e) we plot the number distribution of electrons created via ionization versus the laser pulse envelope position. In Fig. 3(a) with $\omega_{1} / \omega_{0}=2$ there are two ionization points in each cycle of the $\omega_{0}$ laser pulse [36] and tens of ionization points in total can be observed. In Fig. 3(e) with $\omega_{1} / \omega_{0}=1 / 9$, there are six ionization points in each $\omega_{1}$ laser cycle $\tau_{1}=9 \tau_{0}$ (e.g., a cycle from $t-x / c=10 \tau_{0}$ to $19 \tau_{0}$ ) and the total ionization points are less. Note that the total laser field and the current generation is periodic and its period is $b \tau_{0}$ or $a \tau_{1}$, where $\omega_{1} / \omega_{0}=a: b$. In the case with $\omega_{1} / \omega_{0}=2$, the currents formed in each ionization points have the same sign $[17,36]$, as seen in Fig. $3(f)$. In the case with $\omega_{1} / \omega_{0}=1 / 9$, the 
currents formed at the first 3 points and within the first half $\omega_{1}$-laser-cycle (starting around $10 \tau_{0}$ ) have the signs opposite to the ones within the followed half $\omega_{1}$-laser-cycle (starting around $15 \tau_{0}$ ), as observed in Fig. 3(j). According to simple analysis as done in Ref. [36], the currents formed within the first half $\omega_{1}$-laser-cycle should be completely counteracted by the ones within the other half $\omega_{1}$-laser-cycle and $\mathrm{THz}$ radiation should not be generated if the laser pulses have sufficiently large number of cycles [this is the case with $\omega_{1} / \omega_{0}=3$ shown in Figs. 3(d), 3(i) and 3(n)]. However, in our case the laser duration is $50 \mathrm{fs}$ and the laser intensity at the first half $\omega_{1}$-laser-cycle is significantly different from that at the other half $\omega_{1}$-laser-cycle, since the displacement between them is $12 \mathrm{fs}$. Hence, the respective currents formed within the two half cycle cannot be completely counteracted each other, as observed in Fig. 3(j). This causes that the total net current is even stronger than the case with $\omega_{1} / \omega_{0}=2$ [see Fig. 3(o) and $3(\mathrm{k})$ ] and consequently the $\mathrm{THz}$ radiation is more powerful [see Fig. 1]. In the same way, one can explain the powerful $\mathrm{THz}$ generation at other low $\omega_{1} / \omega_{0}$, e.g., $1 / 7,3 / 10,1 / 5$, etc.

The net currents and the $\mathrm{THz}$ radiation are formed in the case with the lower $\omega_{1} / \omega_{0}$ due to a larger period $b \tau_{0}$ or $a \tau_{1}$ of the two linear-superposition laser fields and resulting asymmetry of the laser intensity in the positive and negative half period. Therefore, it is not necessary for $\mathrm{THz}$ generation that the total laser fields are periodic. For example, we take $\omega_{1} / \omega_{0}=0.112(a=112$ and $b=1000)$ in our simulation and a strong radiation can be generated. One can consider the total laser fields of the $50 \mathrm{fs}$ pulses not to be periodic since $b \tau_{0}=a \tau_{1} \gg 50 \mathrm{fs}$. In this case the current and the radiation strength strongly depend on the laser duration, as in the case with $\omega_{1} / \omega_{0}=1 / 9$ [see Fig. 3(o)]. This is different from the case with the higher $\omega_{1} / \omega_{0}$ [e.g., to see Figs. $3(\mathrm{f})-3(\mathrm{~h})$ with $\omega_{1} / \omega_{0}=2,4,8$ ], where the current formation is slightly affected by the change of the laser duration [see Figs. 3(k)-3(m)]. For example in Figs. 3(a) and 3(e), when we decrease the laser duration from $50 \mathrm{fs}$ to $47 \mathrm{fs}$, more electrons are created at a smaller $t-x / c$ and less at a larger $t-x / c$ (at $t-x / c>15 \tau_{0}$ ) because the laser intensity peak is shifted forwards. In the case with $\omega_{1} / \omega_{0}=2$, the total current is nearly not changed [see Fig. 3(k)] since the decrease of the currents at larger $t-x / c$ can be compensated by the increase of the currents at smaller $t-x / c$ [see Fig. 3(f)]. This is not the case with $\omega_{1} / \omega_{0}=1 / 9$. Compared with the case with the 50 fs duration, in the case with the 47 fs duration the positive currents at about $t-x / c=12 \tau_{0}$ and $15 \tau_{0}$ are increased and the negative currents at about $t-x / c=17 \tau_{0}$ and 
$19 \tau_{0}$ are decreased [see Fig. 3(j)] and therefore the total positive current is increased [see Fig. 3(o)].

\section{A. Three series of $\omega_{1} / \omega_{0}$ for high-efficiency and stable THz generation}

From Figs. 1(a) and 1(b) one can also observe that when the laser duration is changed, three series $\omega_{1} / \omega_{0}=2 n, n+1 / 2$, and $n \pm 1 / 3$ ( $n$ is a positive integer) show better stability in the $\mathrm{THz}$ strengths than the cases with lower $\omega_{1} / \omega_{0}$, although in which strong $\mathrm{THz}$ radiation can be generated. In the three series, the periodic current generation has a small period determined by $b \tau_{0}$ (it is $\tau_{0}, 2 \tau_{0}$, and $3 \tau_{0}$ for the series $2 n, n+1 / 2$, and $n \pm 1 / 3$, respectively) and the net currents generated in different periods have the same signs (they could be generated in a few ionization points in each period), as seen in Figs. 3(f)-3(h). A small change of the laser duration (from 50 fs to 47 fs and 40 fs in our simulations) slightly affects the ionization region or the ionization point number and the electron number in each ionization point, as shown in Fig. 3. Therefore, the THz strengths hardly change with the varying laser duration.

In Fig. 2 we increase the laser intensity from $I_{0}=I_{1}=10^{14} \mathrm{~W} / \mathrm{cm}^{2}$ to $4 \times 10^{14} \mathrm{~W} / \mathrm{cm}^{2}$, which causes that the $\mathrm{THz}$ strengths are slightly changed for the $2 n$ and $n+1 / 2$ series, but they are significantly changed for the $n \pm 1 / 3$ series. This can be explained as follows. When the intensity is increased, the ionization points shift away from the intensity peak to the pulse leading edge and the ionization region as well as the ionization point number are reduced, which was demonstrated in Refs. [19,36]. The series $n \pm 1 / 3$ have a longer period $b \tau_{0}(b=3)$ of the periodic current generation than the other two series and therefore, the reduction of the ionization point number affects the net current and $\mathrm{THz}$ generation more significantly. With the same reason, the series $\omega_{1} / \omega_{0}=2 n$ with the shortest period $\tau_{0}$ should have the best stability against the change of the laser intensity.

On the other hand, if the duration is significantly decreased, which causes a significant decrease of the ionization point number, the stability of the series $\omega_{1} / \omega_{0}=2 n$ can become worse. This can be seen from the PIC simulation results in Ref. [19]: with a 12 fs laser duration, the $\mathrm{THz}$ strength shows oscillatory dependence on the laser intensity. By contrast, one can see in Fig. 5 that with the 50 fs laser duration, the $\mathrm{THz}$ strength appears saturation as the laser intensity is increased, i.e., the strength is stable with the increasing laser intensity. 
From Fig. 2 one can also see that the $\mathrm{THz}$ strengths tend to decrease with the growth of $n$ in the three series and the strengths are close to zero with a sufficiently large $n$. With the same $n$, the $2 n$ series have the highest efficiency of $\mathrm{THz}$ generation, e.g., one can compare the THz strengths with $\omega_{1} / \omega_{0}=2,3 / 2$, and $2 / 3$. These results can be explained in the following by a model based on gas ionization by two linear-superposition laser fields and sequently net current or velocity gain. Note that we classify $\omega_{1} / \omega_{0}=1 / 4$ and $1 / 2$ into $2 n$ series. We will show that the analysis to $\omega_{1} / \omega_{0}=4$ and 2 can be directly applied to $\omega_{1} / \omega_{0}=1 / 4$ and $1 / 2$ by a linear scaling law of the laser wavelength derived below.

\section{B. A model and a wavelength scaling law}

The following analysis is based on net or transient current formation and $\mathrm{THz}$ generation via asymmetric ionization of tunneling in two linear-superposition laser fields. The idea was proposed by Kim et al. in 2007 [17] and verified by experiments [17, 18]. Then, it was well established with near-field radiation generation due to the current dynamics in plasma $[19,36,42]$ and far-field radiation obtained by a $(3+1)$-dimensional simulation approach including a comprehensive description of the propagation of lasers and radiation $[40,41]$. Here, we apply the near-field radiation model, since the observed far-field radiation is expected to be composed of near-field radiation sources [27, 41]. Based on the net current formation due to ionization [17], Wang et al. [19] proposed a model including the net current dynamics due to plasma response, which causes the radiation with a frequency at the plasma oscillation frequency; then a solution of the radiation emitted from a plasma was given in a one-dimensional approximation or a plane wave approximation [42]; recently this model has been extended to the case with an external magnetic field imposed [31].

We take the electric fields of the two laser pulses as $\mathbf{E}=\vec{e}_{y}\left[a_{0} \sin \left(\omega_{0} \psi\right)+a_{1} \sin \left(\omega_{1} \psi\right)\right] f(\psi)$, where $\psi=t-x / c$ and $f(\psi)$ is the envelope profile of the laser pulses. We consider the laser duration around 50 fs and thus $\partial f(\psi) / \partial \psi \ll \omega_{0}$ and $\omega_{1}$ in the case $n \geq 1$. Then the vector potential can be given by $\mathbf{A}=\vec{e}_{y} \frac{c}{\omega_{0}}\left[a_{0} \cos \left(\omega_{0} \psi\right)+\frac{\omega_{0}}{\omega_{1}} a_{1} \cos \left(\omega_{1} \psi\right)\right] f(\psi)$. According to Refs. [36], when an electron is born due to ionization, it will gain a net transverse velocity $\mathbf{v}=-e \mathbf{A}\left(\psi^{0}\right) / m_{e} c$ after the passage of the laser pulses, where $\psi^{0}$ is the born position of the electron. Generally $\psi^{0}$ is around an extremum of $E$, at which the ionization occurs with the 
highest probability. An extremum of $E$ appears when $\partial E / \partial \psi=0$, i.e.,

$$
a_{0} \cos \left(\omega_{0} \psi^{0}\right)=-\frac{a_{1} \omega_{1}}{\omega_{0}} \cos \left(\omega_{1} \psi^{0}\right) .
$$

Then, the electron created at $\psi^{0}$ gains the net velocity as

$$
v=\frac{e a_{0}}{m_{e} \omega_{0}} \cos \left(\omega_{0} \psi^{0}\right) f\left(\psi^{0}\right)\left[\frac{\omega_{0}^{2}}{\omega_{1}^{2}}-1\right] .
$$

According to Eqs. (1) and (2), one can derive a scaling law of the laser wavelength under the following conditions. We fix the ratio $\omega_{1} / \omega_{0}$ and the laser field strengthes $a_{0}$ and $a_{1}$ (note that $a_{0}$ can be different from $a_{1}$ ). Equation (1) gives $\cos \left(\omega_{0} \psi^{0}\right)=\alpha \cos \left(\omega_{1} \psi^{0}\right)$, and $\alpha$ is not varied with the change of $\omega_{0}$, and then $\psi^{0}$ is not changed either. If the laser duration is much longer than both the laser cycles $\tau_{0}$ and $\tau_{1}, f\left(\psi^{0}\right)$ will nearly not change with $\omega_{0}$. Hence, properties of the net velocity generation are kept unchanged if one varies $\omega_{0}$ and fixes $\omega_{1} / \omega_{0}$. Then, one can obtain:

$$
v \propto \frac{1}{\omega_{0}} \propto \lambda_{0}
$$

According to the results given in Refs. $[19,31,42]$, the $\mathrm{THz}$ strength $E_{T H z}$ is linearly proportional to the net velocity and therefore the wavelength scaling law for the THz strength can be written by

$$
E_{T H z} \propto \lambda_{0}
$$

Note that Eqs. (3) and (4) hold only when the ionization rates do not depend on the laser wavelength. In this study, we take $I_{0}=I_{1} \geq 10^{14} \mathrm{~W} / \mathrm{cm}^{2}$, the Keldysh parameter [43] $\gamma_{K}$ for hydrogen is less than 1 and therefore the ionization is in the tunneling regime [43, 44]. In this regime, the ionization rates are independent of the wavelength $[45,46]$. When the laser intensity is sufficiently low (or the intensity is depleted due to a long distance propagation [35]), the ionization will enter the multiphoton regime and it will have complex dependence on the wavelength. In this case, the linear scaling of the net current and $\mathrm{THz}$ strength with the wavelength is broken, as shown in Refs. [34, 35].

Equation (1) gives multiple solutions to $\psi^{0}$ and these solutions in the first half period of the total laser field are symmetric to those in the other half period, where a period of the laser field is $\tau_{0}, 2 \tau_{0}, 3 \tau_{0}$, respectively, for the series $\omega_{1} / \omega_{0}=2 n, n+1 / 2$, and $n \pm 1 / 3$. Since the solutions are difficult to derive analytically, we numerically solve Eqs. (1) and (2) and 
TABLE I. Velocities $v$ (normalized by $e a_{0} / m_{e} \omega_{0}$ ) of the electrons created in different ionization points in a half period of the total laser fields and around the laser pulse peak. Note that the same velocity values appear symmetrically in the other half period. The laser frequency ratio is given in the first column and the following columns show the velocities gained from the first, second, and third strongest ionization points determined according to our PIC simulation results, which are partially shown in Figs. 3(f)-3(j). Here, the strongest ionization points mean the ones where the most electrons are created.

\begin{tabular}{lrrr}
\hline \hline & 1st & 2nd & 3nd \\
\hline$\omega_{1} / \omega_{0}=1 / 4$ & 1.36 & -3.38 & \\
$\omega_{1} / \omega_{0}=1 / 2$ & -0.89 & & \\
$\omega_{1} / \omega_{0}=2$ & -0.44 & & \\
$\omega_{1} / \omega_{0}=4$ & 0.34 & -0.87 & \\
$\omega_{1} / \omega_{0}=6$ & -0.25 & 0.67 & \\
$\omega_{1} / \omega_{0}=8$ & 0.19 & -0.54 & 0.88 \\
$\omega_{1} / \omega_{0}=3 / 2$ & -0.20 & 0.50 & \\
$\omega_{1} / \omega_{0}=5 / 2$ & 0.23 & -0.62 & \\
$\omega_{1} / \omega_{0}=2 / 3$ & -0.30 & 0.77 & \\
$\omega_{1} / \omega_{0}=4 / 3$ & -0.18 & 0.30 & -0.43 \\
\hline \hline
\end{tabular}

present the results in Table I. In this table we list the net velocities of the electrons created in the first, second, and third strongest ionization points, where the strongest ionization points mean the ones with the most electrons created. These points are confirmed according to the laser electric field values and our PIC simulation results [partially shown in Figs. 3(f)-3(j)]. Furthermore, we display the net velocities in the strongest ionization points in Figs. 4(a) and $4(\mathrm{~b})$ for the series $\omega_{1} / \omega_{0}=2 n$ and $n+1 / 2$, respectively. The velocities tend to decrease with the increasing $n$ and their signs are changed as $n$ grows by 1, which is in agreement with the simulations results given in Fig. 2. One can also see in Table I that with the same $n$, the $2 n$ series have higher velocities in the strongest ionization points than the other two series, which is in agreement with our PIC simulation shown in Fig. 2.

One can notice that the decrease of the THz strength with $n$ in Fig. 2 is more significant than in Figs. 4(a) and 4(b). This is because the velocities in the second strongest ionization 
points [see Tab. I] have the opposite signs and larger absolute values than the ones in the strongest points (see Table I). Then, the net currents in the strongest points are counteracted [see Figs. 3(g), 3(h)]. With a larger n, e.g., Fig. 3(h), the number of the created electrons in the second (and even the third) strongest point is comparable with the number in the strongest point because the displacement between the two points becomes smaller and the laser fields in these points have smaller difference. Then, the current in the strongest ionization point can be nearly completely counteracted, which causes the total current is weak [see Fig. 3(m)] and consequently the THz generation is ineffective with a large $n$.

In Fig. 4(c) we compare the PIC results with the calculation results of Eq. (2), where we only use the electron velocity in the strongest ionization point. The two results agree with each other for small $n$ since nearly all electrons are created in the strongest ionization point, e.g., $\omega_{1} / \omega_{0}=1 / 2,2,2 / 3$, and $3 / 2$. They appear considerable difference when $n$ becomes large, e.g., $\omega_{1} / \omega_{0}=6$ and $7 / 2$, with which many electrons can be created in the second and third strongest ionization points.

In particular, the results in Fig. 4(c) show good agreement with the linear wavelength scaling law for the THz strength given by Eqs. (3) and (4). One can see the THz strength ratio is $2: 1$ between the cases $\omega_{1} / \omega_{0}=1 / 2$ and $\omega_{1} / \omega_{0}=2$; the strength ratio is $3: 2$ between $\omega_{1} / \omega_{0}=2 / 3$ and $\omega_{1} / \omega_{0}=3 / 2$; and the strength ratio is $4: 1$ between $\omega_{1} / \omega_{0}=1 / 4$ and $\omega_{1} / \omega_{0}=4$. Here we take $I_{1}=I_{0}$ in our simulations (following Ref. [37]) and then can apply Eq. (4) with interchanging the indices 0 and 1 in the frequencies. For example, with $\omega_{1} / \omega_{0}=2$ the laser wavelengths $\lambda_{1}$ and $\lambda_{0}$ are $0.4 \mu \mathrm{m}$ and $0.8 \mu \mathrm{m}$, respectively; with $\omega_{1} / \omega_{0}=1 / 2$ the laser wavelengths $\lambda_{1}$ and $\lambda_{0}$ are $1.6 \mu m$ and $0.8 \mu m$, respectively. With a given frequency ratio $2 / 1$, the $1.6 \mu \mathrm{m}$ laser is the fundamental one in the latter case, while in the former case the $0.8 \mu \mathrm{m}$ laser is the fundamental one. Therefore, as the fundamental wavelength is changed by a factor of 2 , the $\mathrm{THz}$ strength should change by the same factor according to the wavelength scaling law.

If $I_{0}$ and $I_{1}$ are different, the linear wavelength scaling is still valid with a fixed frequency ratio, since the tunneling ionization rates depend on the total laser intensity and does not depend on the wavelength. In Fig. 5, we show the PIC simulation results with $I_{1}=0.25 I_{0}$ and one can see the linear scaling holds well. Note that the scaling law $E_{T H z} \propto \lambda_{0}$ or $\varepsilon_{T H z} \propto \lambda_{0}^{2}\left(\varepsilon_{T H z}\right.$ is the THz energy) disagrees with the scaling law $\varepsilon_{T H z} \propto \lambda_{0}^{4.6}$ found in experiments [34]. There are two reasons at least. First, we fix the laser intensity, duration, 
and spot size as the wavelength is varied. In Ref. [34] the laser energy was fixed with the varying wavelength, which results in the change of the laser intensity and laser-gas or laser-plasma interaction volume. Second, our scaling law is given in the tunneling ionization regime in which the ionization rates do not depend on the wavelength. In Ref. [34] the ionization could stride across the tunneling regime and the multiphoton ionization regime as the laser wavelength/intensity is changed. In the multiphoton regime, the ionization rates depend on the wavelength. The two factors above cause a stronger dependence of the $\mathrm{THz}$ energy on the wavelength.

\section{SUMMARY}

In summary, we have investigated $\mathrm{THz}$ generation via the two-color laser scheme with the frequency ratio $\omega_{1} / \omega_{0}$ in the nearly whole range possibly adopted in laboratories currently. For a conventional $0.8 \mu \mathrm{m}$ and 50 fs laser pulse (the $\omega_{0}$ pulse), THz radiation may be generated at any $\omega_{1} / \omega_{0}$ when $\omega_{1} / \omega_{0}<1 / 3$. Even at $\omega_{1} / \omega_{0}=a / b=1 / 5,1 / 7$ with an even $a+b$, the radiation can be generated and its strength could be higher than the one at

$\omega_{1} / \omega_{0}=2$, the original two-color scheme. In the case with the lower $\omega_{1} / \omega_{0}$, the net current is formed due to the asymmetry of the laser intensity in the positive and negative half period of the laser fields. Therefore, the $\mathrm{THz}$ strength is sensitive to the laser pulse duration and periodic laser fields are not necessary for the $\mathrm{THz}$ generation.

With $\omega_{1} / \omega_{0}>1 / 3$, there are three series with $\omega_{1} / \omega_{0}=2 n, n+1 / 2, n \pm 1 / 3$ for highefficiency and stable $\mathrm{THz}$ generation, where periodic laser fields are necessary to ensure that net currents formed in different periods have the same sign. Among them, the $2 n$ series shows the highest efficiency and the best stability against the change of the laser duration and intensity. For the three series the $\mathrm{THz}$ strength basically decreases with the increasing $n$ and the strength scales linearly with the laser wavelength. The linear scaling holds in the tunneling ionization regime.

\section{ACKNOWLEDGMENTS}

This work was supported by the National Basic Research Program of China (Grant No. 2013CBA01500 and 2014CB339801), the National Natural Science Foundation of China 
(Grants No. 11375261, No. 11421064, No. 11374210, and No. 113111048), Science Challenge Project of China (Grant No. TZ2016005), and the Strategic Priority Research Program of the Chinese Academy of Sciences (Grants No. XDB16010200 and XDB07030300). Z.M.S. acknowledges the support of a Leverhulme Trust Research Grant and EPSRC (UK) Grant No. EP/N028694/1.

[1] D. Grischkowsky, S. Keiding, M. Exter, and C. Fattinger, J. Opt. Soc. Am. B 7, 2006 (1990).

[2] J. C. Cao, Phys. Rev. Lett. 91, 237401 (2003).

[3] M. Jewariya, M. Nagai, and K. Tanaka, Phys. Rev. Lett. 105, 203003 (2010).

[4] T. Kampfrath, K. Tanaka, and K. Nelson, Nat. Photonics 7, 680 (2013).

[5] B. Alexandrov, M. Phipps, L. Alexandrov, L. Booshehri, A. Erat, J. Zabolotny, C. Mielke, H.-T. Chen, G. Rodriguez, K. Rasmussen, J. S. Martinez, A. R. Bishop, and A. Usheva, Sci. Rep. 3, 1184 (2013).

[6] R. Woodward, V. Wallace, R. Pye, B. Cole, D. Arnone, E. Linfield, and M. Pepper, J. Invest. Dermatol. 120, 72 (2003).

[7] Y. T. Li, C. Li, M. L. Zhou, W. M. Wang, F. Du, W. J. Ding, X. X. Lin, F. Liu, Z. M. Sheng, X. Y. Peng et al., Appl. Phys. Lett. 100, 254101 (2012).

[8] A. Gopal, S. Herzer, A. Schmidt, P. Singh, A. Reinhard, W. Ziegler, D. Brommel, A. Karmakar, P. Gibbon, U. Dillner et al., Phys. Rev. Lett. 111, 074802 (2013).

[9] G. Q. Liao, Y. T. Li, C. Li, L. N. Su, Y. Zheng, M. Liu, W. M. Wang, Z. D. Hu, W. C. Yan, J. Dunn, J. Nilsen, J. Hunter, Y. Liu, X. Wang, L. M. Chen, J. L. Ma, X. Lu, Z. Jin, R. Kodama, Z. M. Sheng, and J. Zhang, Phys. Rev. Lett. 114, 255001 (2015).

[10] G.-Q. Liao, Y.-T. Li, Y.-H. Zhang, H. Liu, X.-L. Ge, S. Yang, W.-Q. Wei, X.-H. Yuan, Y.-Q. Deng, B.-J. Zhu, Z. Zhang, W.-M. Wang, Z.-M. Sheng, L.-M. Chen, X. Lu, J.-L. Ma, X. Wang, and J. Zhang, Phys. Rev. Lett. 116, 205003 (2016).

[11] W. J. Ding and Z. M. Sheng, Phys. Rev. E 93, 063204 (2016).

[12] Z. Jin, H. B. Zhuo, T. Nakazawa, J. H. Shin, S. Wakamatsu, N. Yugami, T. Hosokai, D. B. Zou, M. Y. Yu, Z. M. Sheng, and R. Kodama Phys. Rev. E 94, 033206 (2016).

[13] D. J. Cook and R. M. Hochstrasser, Opt. Lett. 25, 1210 (2000).

[14] M. Kress, T. Loffler, S. Eden, M. Thomson, and H. G. Roskos, Opt. Lett. 29, 1120 (2004). 
[15] T. Bartel, P. Gaal, K. Reimann, M. Woerner, and T. Elsaesser, Opt. Lett. 30, 2805 (2005).

[16] X. Xie, J. Dai, and X.-C. Zhang, Phys. Rev. Lett. 96, 075005 (2006).

[17] K. Y. Kim, J. H. Glownia, A. J. Taylor and G. Rodriguez, Opt. Express 15, 4577 (2007).

[18] K. Y. Kim, A. J. Taylor, J. H. Glownia, and G. Rodriguez, Nat. Photonics 2, 605 (2008).

[19] W.-M. Wang, Z.-M. Sheng, H.-C. Wu, M. Chen, C. Li, J. Zhang, and K. Mima, Opt. Express 16, 16999 (2008).

[20] X.-Y. Peng, C. Li, M. Chen, T. Toncian, R. Jung, O. Willi, Y.-T. Li, W.-M. Wang, S.-J. Wang, F. Liu, A. Pukhov, Z.-M. Sheng, and J. Zhang, Appl. Phys. Lett. 94, 101502 (2009).

[21] T.-J. Wang, Y. Chen, C. Marceau, F. Theberge, M. Chateauneuf, J. Dubois, and S. L. Chin, Appl. Phys. Lett. 95, 131108 (2009).

[22] Y. Chen, T.-J. Wang, C. Marceau, F. Theberge, M. Chateauneuf, J. Dubois, O. Kosareva and S. L. Chin, Appl. Phys. Lett. 95, 101101 (2009).

[23] W.-M. Wang, P. Gibbon, Z.-M. Sheng, and Y.-T. Li, Phys. Rev. A 90, 023808 (2014).

[24] A. Debayle, P. Gonzalez de Alaiza Martinez, L. Gremillet, and L. Berge, Phys. Rev. A 91, $041801(2015)$.

[25] P. Gonzalez de Alaiza Martinez, X. Davoine, A. Debayle, L. Gremillet, and L. Berge, Sci. Rep. 6, 26743 (2016).

[26] V. A. Andreeva, O. G. Kosareva, N. A. Panov, D. E. Shipilo, P. M. Solyankin, M. N. Esaulkov, P. Gonzalez de Alaiza Martinez, A. P. Shkurinov, V. A. Makarov, L. Berge, and S. L. Chin, Phys. Rev. Lett. 116, 063902 (2016).

[27] Z. Zhang, Y. Chen, M. Chen, Z. Zhang, J. Yu, Z. Sheng, J. Zhang, Phys. Rev. Lett. 117, 243901 (2016).

[28] H. C. Wu, J. Meyer-ter-Vehn, and Z. M. Sheng, New J. Phys. 10, 043001 (2008).

[29] J. Dai, N. Karpowicz, and X.-C. Zhang, Phys. Rev. Lett. 103, 023001 (2009).

[30] H. Wen and A. M. Lindenberg, Phys. Rev. Lett. 103, 023902 (2009).

[31] W.-M. Wang, P. Gibbon, Z.-M. Sheng, and Y.-T. Li, Phys. Rev. Lett. 114, 253901 (2015).

[32] W.-M. Wang, S. Kawata, Z.-M. Sheng, Y.-T. Li, J. Zhang, L. M. Chen, L. J. Qian, J. Zhang, Opt. Lett. 36, 2608 (2011).

[33] Y. Bai, L. Song, R. Xu, C. Li, P. Liu, Z. Zeng, Z. Zhang, H. Lu, R. Li, and Z. Xu, Phys. Rev. Lett. 108, 255004 (2012).

[34] M. Clerici, M. Peccianti, B. E. Schmidt, L. Caspani, M. Shalaby, M. Giguere, A. Lotti, A. 
Couairon, F. Legare, T. Ozaki, D. Faccio, and R. Morandotti, Phys. Rev. Lett. 110, 253901 (2013).

[35] L. Berge, S. Skupin, C. Kohler, I. Babushkin, and J. Herrmann, Phys. Rev. Lett. 110, 073901 (2013).

[36] W.-M. Wang, Y.-T. Li, Z.-M. Sheng, X. Lu, and J. Zhang, Phys. Rev. E 87, 033108 (2013).

[37] V. A. Kostin, I. D. Laryushin, A. A. Silaev, and N. V. Vvedenskii, Phys. Rev. Lett. 117, $035003(2016)$.

[38] P. Gonzalez de Alaiza Martinez, I. Babushkin, L. Berge, S. Skupin, E. Cabrera-Granado, C. Kohler, U. Morgner, A. Husakou, and J. Herrmann, Phys. Rev. Lett. 114, 183901 (2015).

[39] W.-M. Wang, P. Gibbon, Z.-M. Sheng, and Y.-T. Li, Phys. Rev. E 91, 013101 (2015).

[40] I. Babushkin, W. Kuehn, C. Kohler, S. Skupin, L. Berge, K. Reimann, M. Woerner, J. Herrmann, and T. Elsaesser, Phys. Rev. Lett. 105, 053903 (2010).

[41] I Babushkin, S Skupin, A Husakou, C Kohler, E Cabrera-Granado, L Berge and J Herrmann, New J. Phys. 13, 123029 (2011).

[42] W.-M. Wang, S. Kawata, Z.-M. Sheng, Y.-T. Li, and J. Zhang, Phys. Plasmas 18, 073108 (2011).

[43] L. V Keldysh, Soy. Phys. JETP, 20, 1307 (1965).

[44] G. Gibson, T. S. Luk, and C. K. Rhodes, Phys. Rev. A 41, 5049 (1990).

[45] M. V. Ammosov, N. B. Delone, and V. P. Krainov, Sov. Phys. JETP 64, 1191 (1986).

[46] B. M. Penetrante and J. N. Bardsley, Phys. Rev. A 43, 3100 (1991). 


\section{Figure Captions}

Fig. 1. The THz field strength as a function of the two-color laser frequency ratio $\omega_{1} / \omega_{0}$, where different curves correspond to different laser durations. (a) The absolute values of the THz strengths. To clearly show the strengths at low $\omega_{1} / \omega_{0}$, a limited range of $\omega_{1} / \omega_{0}$ is taken in (b).

Fig. 2. The THz field strength as a function of the two-color laser frequency ratio, where the two curves in each plot correspond to laser intensities of $10^{14} \mathrm{~W} / \mathrm{cm}^{2}$ and $4 \times 10^{14} \mathrm{~W} / \mathrm{cm}^{2}$, respectively.

Fig. 3. (a)-(e) The number distribution of electrons as a function of laser pulse envelope position $(t-x / c)$, where the pulse front is at $t-x / c=0$ and the electrons are created via ionization by the two-color laser pulses with different frequency ratios in different plots. The two curves in each plot correspond to laser durations of $50 \mathrm{fs}$ and $47 \mathrm{fs}$, respectively. (f)-(j) The corresponding currents $-J\left(J=-e n_{e} v\right)$ formed due to the ionization, where in each plot the cyan dash line shows the normalized velocities $v$ or the normalized vector potentials $-A$ of the 50 fs two-color laser pulses. (k)-(o) The corresponding total currents after the passage of the two-color laser pulses with durations $50 \mathrm{fs}$ and $47 \mathrm{fs}$, where the colors of the bars display the current signs.

Fig. 4. (a), (b) Velocities $v$ (normalized by $e a_{0} / m_{e} \omega_{0}$ ) of the electrons created in the strongest ionization points, calculated according to Eq. (2), where the frequency ratios are taken as $2 n$ and $n+1 / 2$ in (a) and (b), respectively. (c) The THz field strength as a function of the laser frequency ratio, where the red stars show PIC results and the blue circles show calculation results. The calculation results are fitted through $E_{T H z} / v$ at $\omega_{1} / \omega_{0}=2$ $\left[E_{T H z}\left(\omega_{1} / \omega_{0}=2\right)\right.$ is obtained from our PIC result and $v\left(\omega_{1} / \omega_{0}=2\right)$ from Eq. (2)].

Fig. 5. The THz field strength as a function of the $\omega_{0}$-laser intensity $I_{0}$, where in the PIC simulations we fix $\omega_{1} / \omega_{0}=2$ and $I_{1} / I_{0}=0.25$. We perform two groups of simulations with different $\omega_{0}$-laser wavelengths of $1.6 \mu \mathrm{m}$ and $0.8 \mu \mathrm{m}$ shown by the blue circles and the red crosses, respectively, where the $\mathrm{THz}$ strengths are multiplied by a factor of 2 in the 
case with the wavelength of $0.8 \mu \mathrm{m}$. 
Figure 1:

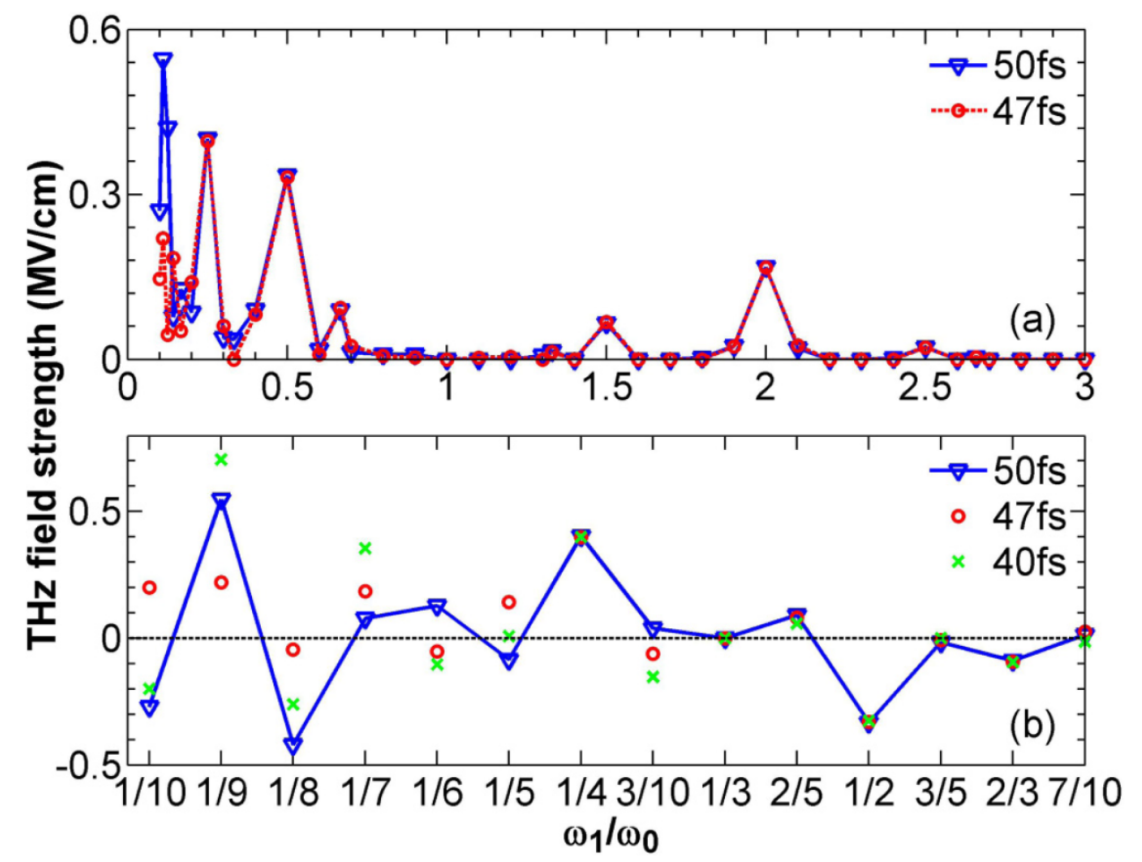

Figure 2:

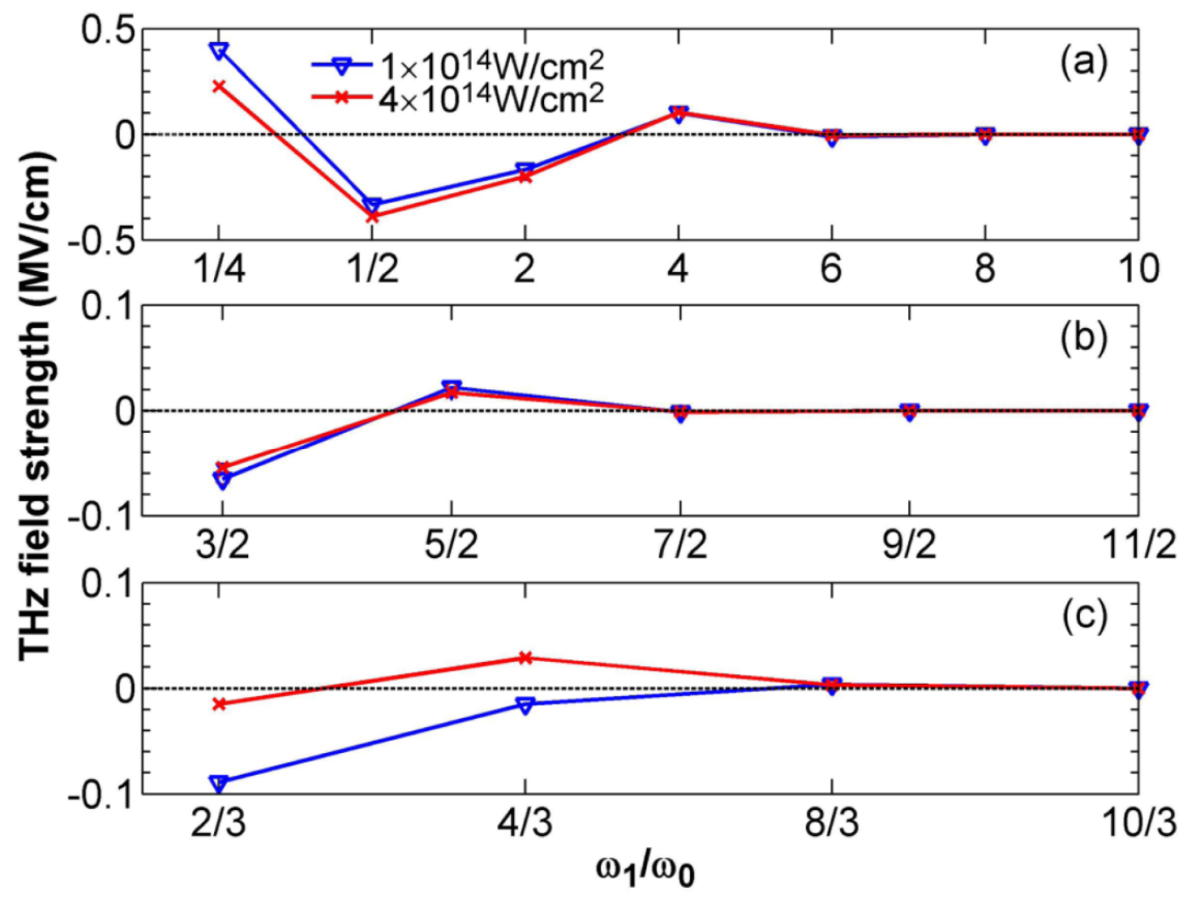


Figure 3:
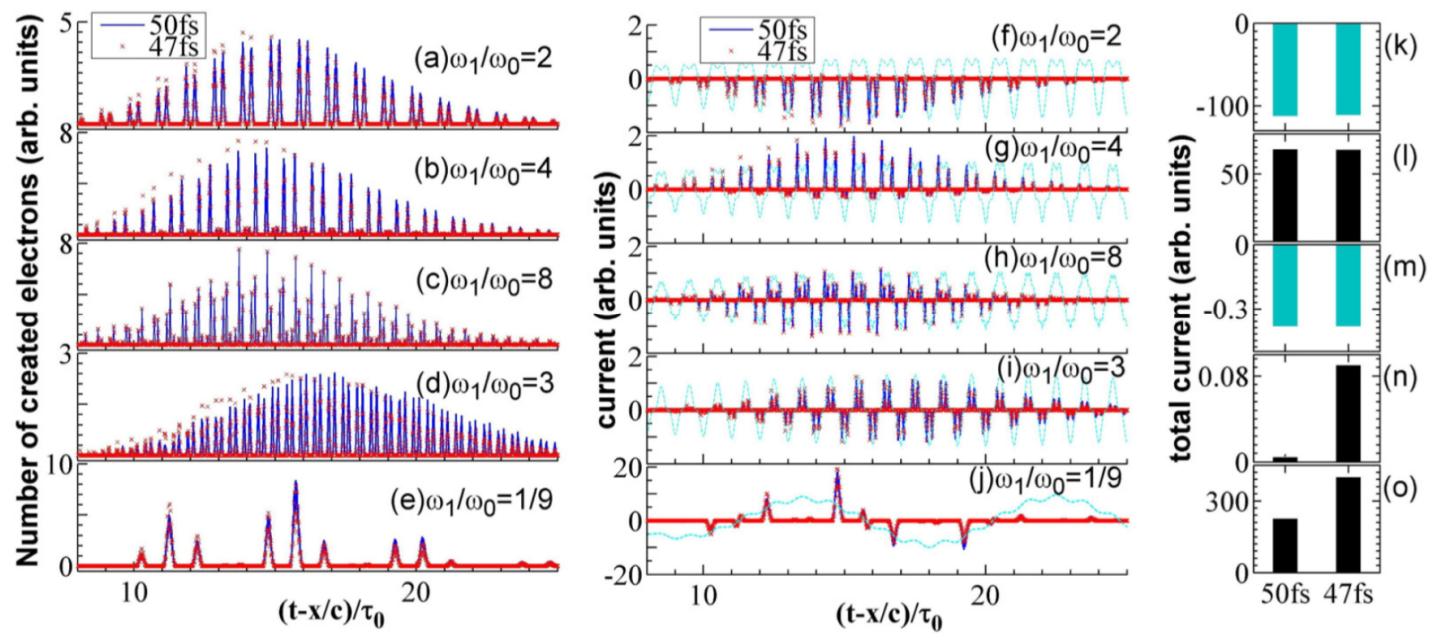

Figure 4:

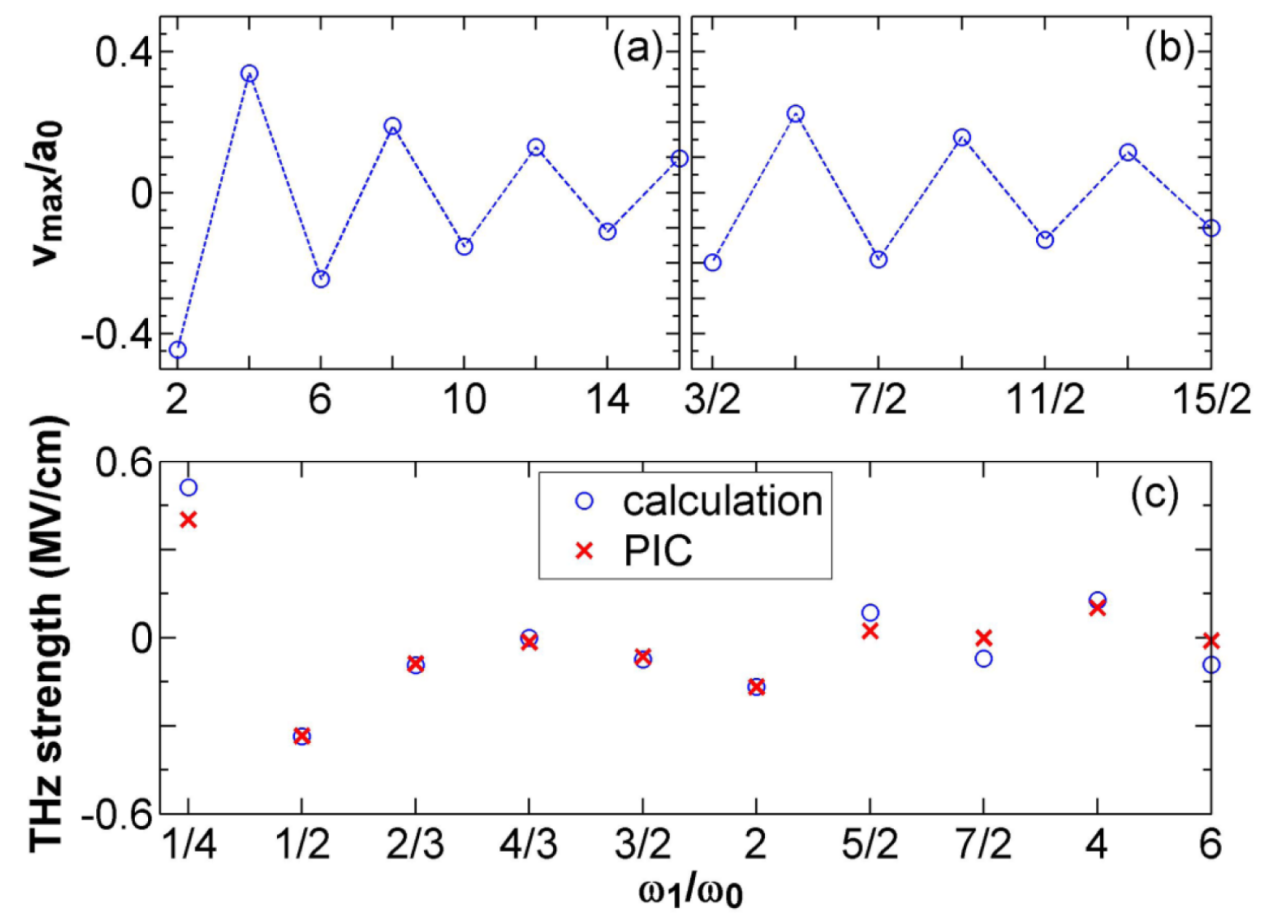


Figure 5:

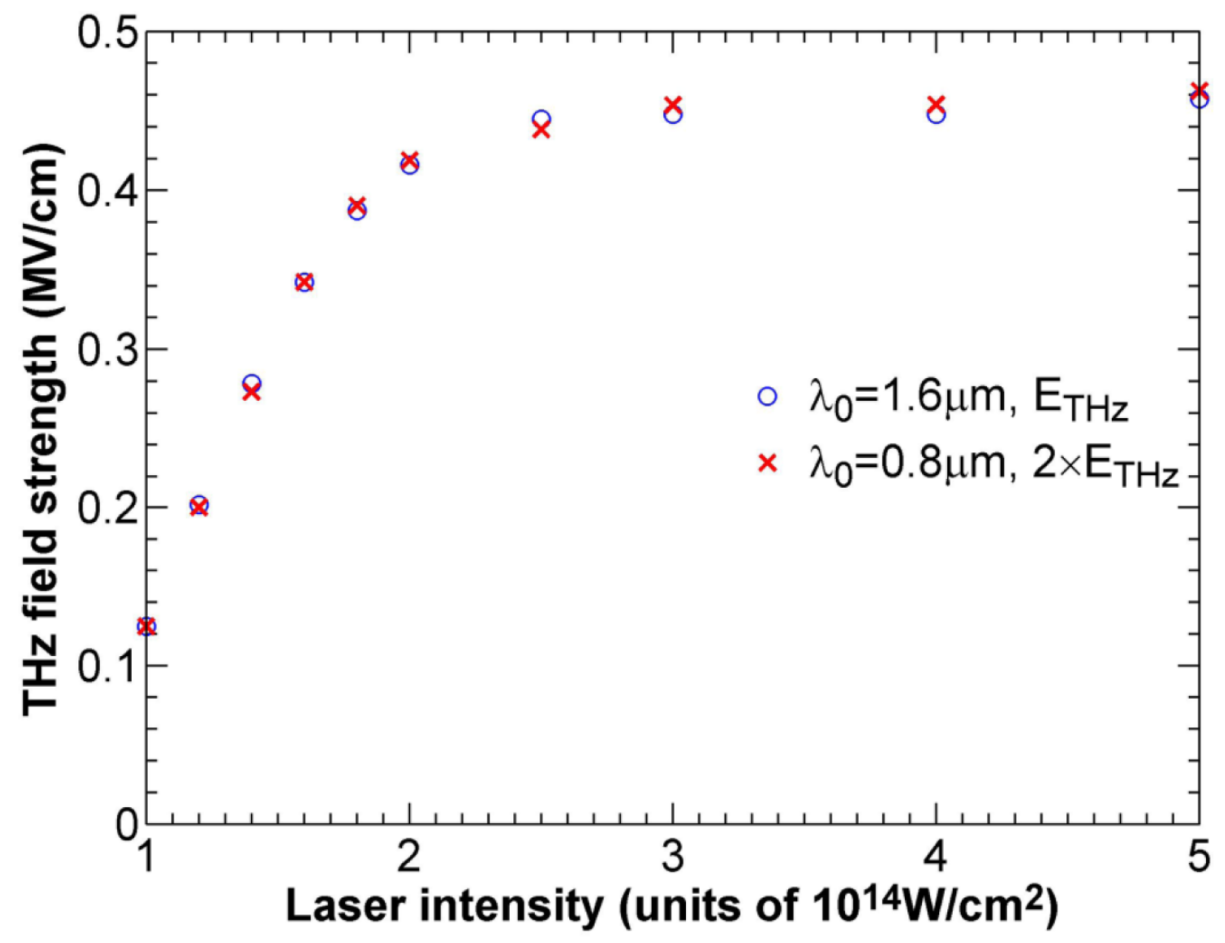

